\title{
Breaking the degeneracy of nematic liquid crystals by means of actu- ated anisometric paramagnetic colloids ${ }^{\dagger}$
}

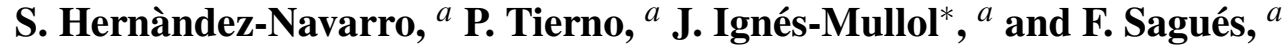 \\ Received Xth $X X X X X X X X X X 20 X X$, Accepted Xth $X X X X X X X X X 20 X X$ \\ First published on the web $X$ th $X X X X X X X X X X 200 X$ \\ DOI: 10.1039/b000000x
}

We demonstrate experimentally that the director of a planar nematic liquid crystal cell with degenerated azimuth can be resolved by means of sparse paramagnetic inclusions actuated upon by weak magnetic fields. The use of ellipsoidal particles enables to characterize the reorientation of the mesogen following particle rotation by means of a simple elastic model. Complex structures like targets and double-armed helices can be generated and controlled by tuning an out-of-plane electric field.

Soft matter is intrinsically labile and thus addressable by means of an appropriate external forcing. However this potentiality is useless without guaranteeing control over the elicited responses, singularly when the latter are prone to be highly degenerated. Indeed this can be the case when referring to two of the most studied categories of soft materials: liquid crystals and colloids. The interest to combine both materials has raised enormously during these last years. Dispersions of colloids in liquid crystals constitute nowadays familiar mixtures sought to unveil new fundamental concepts and original applications ${ }^{1-4}$. Singularly, the capability of the host elastic matrix, with its intrinsic anisotropies, to mediate interactions between suspended colloids was already discovered a long time ago $^{5-8}$ and has been profusely used since then ${ }^{9-12}$. In this respect, novel colloidal structures have been observed experimentally ${ }^{13-17}$.

In contrast to research on liquid crystal dispersions of submicron or nano-scale particles ${ }^{18,19}$, we concentrate here on inclusions at the micrometer scale, which enable a discrete and local control of the medium. Commonly, the dispersed phase consists of spherical particles ${ }^{20}$. More recently, the field has been extended to incorporate anisometric, either actuated $^{21,22}$ or non-actuated ${ }^{23,24}$, inclusions. Tailoring particle shape opens new possibilities regarding the manipulation and assembling of the dispersed entities ${ }^{25,26}$.

\footnotetext{
$\dagger$ Electronic Supplementary Information (ESI) available: [details of any supplementary information available should be included here]. See DOI: 10.1039/b000000x/

a Departament de Química Física and IN2UB, Universitat de Barcelona, Martí i Franquès 1, 08028 Barcelona, Spain. Fax: +34 934021231; Tel: +34 934021220; E-mail: jignes@ub.edu
}

In spite of the extensive research mentioned so far, there is still a non-explored niche which consists in using nonspherical paramagnetic inclusions to control the orientation of a liquid crystal. Our approach is contrary to the usually adopted one, since here externally actuated particles are acting on their host rather than passively adapting to the constraints imposed by the liquid crystal medium where they reside.

As a proof of concept we demonstrate that anisotropic aggregates of paramagnetic spherical particles or isolated paramagnetic ellipsoids, rotated by weak magnetic fields, break the orientational degeneracy of a thin nematic sample when it is transited from homeotropic to planar alignment. In particular, we use a combination of an electric (acting over the medium) and a magnetic field (acting on the inclusions) to orient a liquid crystal material over localized areas around the dispersed colloids. Moreover, we show that with our method it is possible to generate and control different patterns, such as targets and helices.

Nematic liquid crystal (NLC) cells were prepared by using two ITO-glass plates, previously cleaned with a Micro-90 (Sigma-Aldrich) solution. They were coated with a self assembled monolayer of DMOAP ([3(trimethoxysilyl)propyl] octadecyldimethylammonium chloride) to create a homeotropic alignment ${ }^{27}$. Briefly, $0.25 \mathrm{~mL}$ of DMOAP (Aldrich Chemistry) were mixed with $50 \mathrm{~mL}$ of Milli-Q water, then the plates were submerged in the DMOAP solution for 15 minutes, slowly rinsed, dried under a stream of argon and baked at $130^{\circ} \mathrm{C}$ for 2 hours. The two plates, separated by a Mylar spacer, were then glued together. The cell's actual thickness was measured using an interferometric technique. Filling these cells with a NLC characterized by a negative dielectric anisotropy $\left(\varepsilon_{a}\right)$ and applying an a.c. $(1 \mathrm{kHz})$ electric field between the electrodes makes it possible to induce a planar alignment with azimuth degeneration $^{28}$.

We used paramagnetic polystyrene spherical particles (micromer-M PEG-COOH, size 6 and $12 \mu \mathrm{m}$ ), either as received or changing their shape to ellipsoidal, as described elsewhere $^{29}$. Typical long axis of the ellipsoids ranges from 24 to $29 \mu \mathrm{m}$. After evaporating a few drops of the aqueous suspen- 

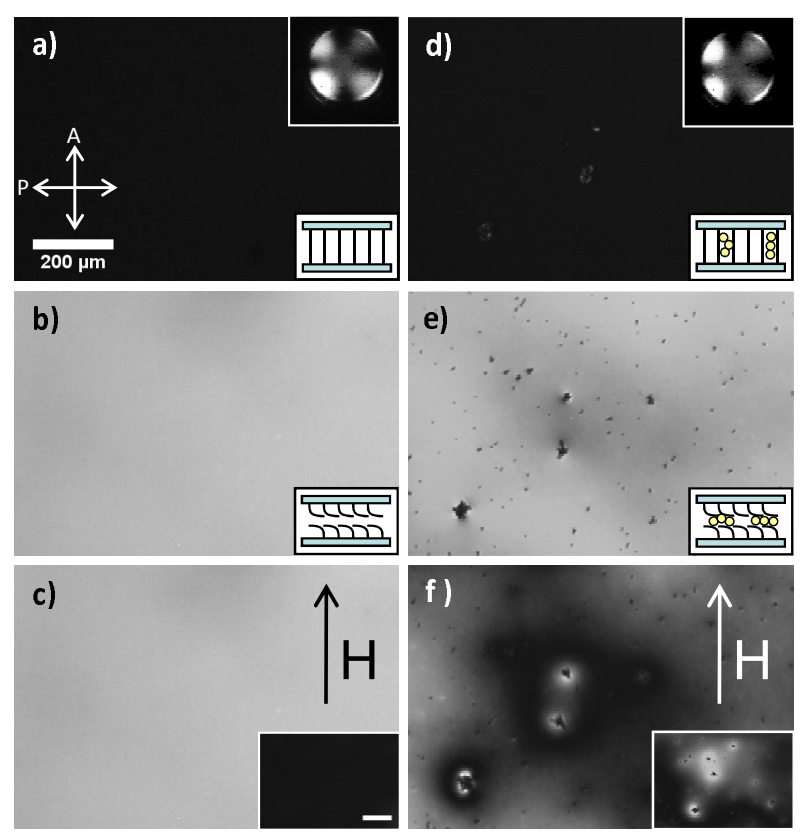

Fig. 1 Polarizing microscope images showing the effect of the paramagnetic inclusions on the NLC orientation. (a), (b), and (c) are images of a NLC cell without particles, and (d), (e), and (f) are images of a NLC cell doped with particles. In the absence of external fields, (a) and (d), the cells are homeotropic (conoscopy image in the inset). An electric field perpendicular to the cell plates is applied in (b), and (e), and an additional in-plane magnetic field $(6 \mathrm{mT})$ is applied in (c) and (f). The inset in the latter frames corresponds to the samples rotated $30^{\circ} \mathrm{CCW}$. Schemes drawn at the right bottom part of images (a), (b), (d) and (e) are representations of a cross section of the cells. Cell thickness is $30 \pm 2 \mu \mathrm{m}$.

sion of microparticles on a glass plate, they were dispersed in one drop of the nematic MLC-7029 $\left(\varepsilon_{a}=-3.6\right.$, Merck) by manual stirring for 1 minute ${ }^{30}$. Finally, the NLC cell was filled with the dispersion by capillarity. According to preliminary observation, the NLC adopts a strong parallel anchoring at the contact surface with the inclusions. Most of our measures were made at 9V, well above the threshold of the Frederiks transition, to maximize the tilt angle of the director.

The experiments were performed with a Polarizing Microscope (Nikon Eclipse 50iPol) equipped with a Helmholtz coil configuration in a custom-built assembly, which enabled us to apply a magnetic field in the in-plane direction and rotate the field in this plane. The potential difference was applied to the cells by using a NI-DAQ board (NI PCI-6221) and it was computer-controlled with a LabView custom-made program. Images were captured with an AVT Marlin F-131C CMOS camera controlled with the software AVT SmartView 1.10.2. Images were then treated and analyzed using the software packages ImageJ and IgorPro.
To better illustrate the reported phenomenon, we start with a homeotropically aligned nematic sample (Fig. 1a) free of paramagnetic inclusions and we first simply apply a ramped electric field parallel to the direction of molecular alignment. The material, having a negative dielectric anisotropy, globally reorients and, above the (electric) Freederiks threshold, progressively bends towards the center of the cell, adopting a planar configuration at its mid plane (Fig. 1b). The ramp is applied slowly $(2 \mathrm{mV} / \mathrm{s})$ to avoid the formation of point defects and Schlieren textures ${ }^{31}$. Although the panel reproduces a small area with a quite uniform reflectivity, larger observed regions display different gray levels, featuring degenerated inplane molecular orientations. Introduction of the magnetic field has no appreciable effect (Fig. 1c).

The experiment is repeated when the cell is filled with the NLC containing paramagnetic spherical particles. The latter, once dispersed in the NLC, organize into separated clusters due to attractive long-range interactions of elastic nature ${ }^{6}$. We observe that once formed, these clusters, which contain up to 10 particles and have anisotropic shape, remain dispersed in the medium over time. In absence of external fields, the clusters align so as to minimize the distortion caused in the host matrix (Fig. 1d). When applying the electric field there is no significant difference (Fig. 1e) with the particle-free sample, since the orientation of the magnetic inclusions is dictated by the degenerated orientation of the NLC. The active role of the inclusions is revealed only after the additional magnetic field is applied on the particle-ladden planar sample. Paramagnetic clusters align along the direction imposed by the magnetic field, which results in a neat and contrasted reflectivity change in areas around the dispersed clusters (Fig. 1f). This clearly shows that the inclusions, when oriented by the magnetic field, are capable to align the NLC director in their neighbourhood. Notice that to be able to transmit a mechanical torque through coupling with the magnetic field the paramagnetic objects must be anisotropic; isolated spherical particles are not affected by the magnetic forcing.

In order to better characterize the particle-induced director reorientation, we analyze the effect of an isolated paramagnetic ellipsoidal particle embedded in the NLC (Fig. 2). The direction of the magnetic field is adjusted so that the reference orientation is given by the local (in-plane) azimuth of the NLC. Modest reorientations induced by slowly rotating the magnetic field result in a dark halo that grows away from the torqued ellipsoid. The maximal effect is realized for a particle reorientation near $90^{\circ}$ (Fig. 2b). Beyond that value, a white halo starts to grow (Fig. 2c) and further rotating the magnetic field leads to a target-like structure (Fig. 2d). Notice that the distortion in the nematic field has rotational symmetry despite being generated by anisotropic inclusions. The effect is reversible and the whole structure is relaxed after turning off the magnetic field (see video in Supplementary Information). The 

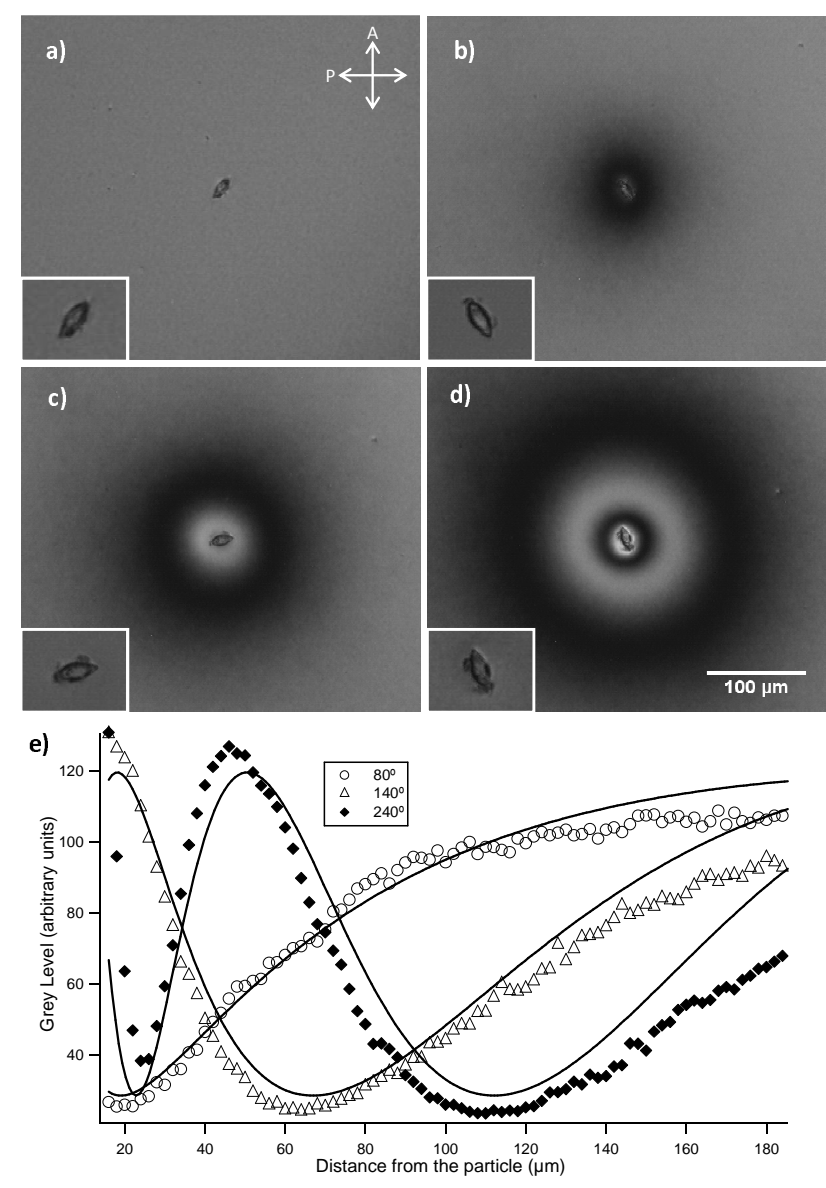

Fig. 2 Distortion generated by a $25 \mu \mathrm{m}$ long, $10 \mu \mathrm{m}$ wide ellipsoid rotated CCW inside a $30 \pm 2 \mu \mathrm{m}$ thick cell. The four insets are enlargements (factor 1.8) of the central ellipsoid. Initial (field-free) orientation is taken as $0^{\circ}$ (a). The magnetic field $(3 \mathrm{mT})$ is applied in (a), and then particle rotates, following the magnetic field, by an angle of $80^{\circ}$ (b), $140^{\circ}$ (c), and $240^{\circ}$ (d). Grey level of the images vs. distance from the particles (e). Solid lines are fits to eq. 3, yielding $r_{0}=2.8 \pm 0.1 \mu \mathrm{m}$, and $R=251 \pm 2 \mu \mathrm{m}$.

inner rings collapse successively towards the central magnetic inclusion as the particle turns back to its original orientation.

A simple model is proposed to account quantitatively for the observed reflectivity pattern around an isolated ellipsoid. Similar modeling approaches have been applied to forced freestanding Smectic-C films ${ }^{32,33}$. We start with the usual oneconstant approximation to express the elastic free energy as

$$
F_{e l}=\int \frac{1}{2} K\left(\partial_{\alpha} n_{\beta} \partial_{\alpha} n_{\beta}\right) d \vec{r}
$$

where $K$ accounts for an average elastic constant, and $\vec{n}=$ $(\cos \phi, \sin \phi, 0)$. A two-dimensional approach is here justified for symmetry reasons and this permits to write the above free energy density in terms of the azimuthal angle $\phi(\vec{r})$ :

$$
F_{e l}=\int \frac{1}{2} K(\nabla \phi)^{2} d \vec{r}
$$

Minimizing eq. (2) under steady state conditions leads to the Laplace equation for in-plane distortions, $\Delta \phi=0$. This harmonic approximation is easily solved using polar coordinates in terms of close- and far-field boundary conditions $\phi\left(r=r_{0}\right)=\phi_{p}, \phi(r=R)=\phi_{b}$. The first condition refers to the distance to the ring center at which the director field has rotated the same angle as the ellipsoid $\left(\phi_{p}\right)$. It must be understood as an effective distance, that should scale with the ellipsoid size. The second prescription refers to the spontaneous azimuth alignment that spans submillimeter regions in the planar NLC configuration. We attribute this phenomenon to the unavoidable presence of orientational quenched disorder on the confining surfaces ${ }^{34}$ and, even though it results in weak bulk forces that are easily overcome by the applied magnetic torques, it nevertheless provides a fixed boundary condition far from the colloidal inclusions. We take into account such effect by considering that at some cut-off distance $\mathrm{R}$ from the center of the analyzed particle, the orientation of the liquid crystal is unaffected by the presence of the ellipsoid, and it keeps its original local value $\phi_{b}$. The latter is taken equal to zero according to the initial condition of the experiment as previously mentioned. The closed solution of eq. (2) then reads:

$$
\phi(r)=\frac{\phi_{p} \ln \left(\frac{r}{R}\right)}{\ln \left(\frac{r_{0}}{R}\right)} .
$$

Here, $\phi_{p}$ is taken as the orientation of the long axis of the ellipsoid, while the parameters $\mathrm{R}$ and $r_{0}$ are fitted to the data (Fig. 2e). Eq. 3 actually corresponds to the "magic spiral" described by de Gennes ${ }^{31}$.

One can readily check, by analyzing Eq. (3), that the director field lines in the target pattern are spirals that wind according to the handedness of the magnetic field rotation ${ }^{35}$. Nevertheless, textures generated by $\mathrm{CW}$ or $\mathrm{CCW}$ rotation have rotational symmetry, and are indistinguishable under the microscope as a result of the planar configuration of the nematic director. We can force the latter to escape into the third dimension by tuning the voltage applied across the sample, bringing its amplitude closer to the electric Frederiks threshold. A few tenths of volts before the particle-induced pattern fades, a helical texture can be observed (Fig. 3). Its handedness correlates with the rotation of the magnetic field used to create the target pattern. The rotational symmetry present in the textures around isolated ellipsoids is now broken, and the anisometric nature of the inclusions is now playing a relevant role.

In this communication we have demonstrated that paramagnetic colloidal inclusions in a nematic liquid crystal can play 

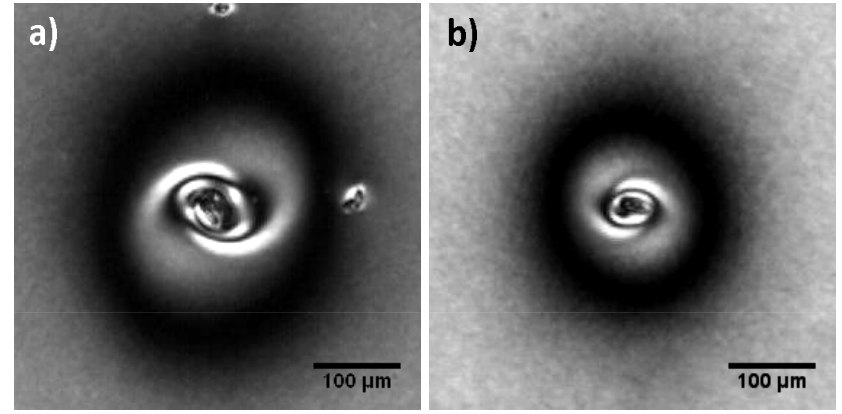

Fig. 3 Polarizing microscope images of double-armed helical patterns under crossed polarizers. These chiral structures appear when the electric field is decreased until $2.5 \mathrm{~V}$ from $9 \mathrm{~V}$. It is possible to distinguish whether the inclusion has been rotated $\mathrm{CW}$ (a) or CCW (b). The corresponding videos are deposited in the Supporting Information.

an active role in the control of the bulk configuration of the mesogen. This constitutes a new paradigm in the interplay between colloids and liquid crystals. The use of anisotropic aggregates or anisometric paramagnetic particles enables to apply a local torque by means of weak magnetic fields, provided the nematic director is not bound by strong anchoring conditions at the cell plates. This is achieved in the present work by means of a planar degenerated configuration obtained with an external electric field, but one could think of other arrangements with gliding anchoring where the colloidal forces would be able to overcome the elastic energy barriers necessary to modify the orientation of a bulk liquid crystal. We have shown that the same technique enables to create stable complex patterns such as targets or chiral textures as an example of the richness of structures that one can envisage by combining two independent orienting external fields, one acting on colloidal inclusions capable of reorienting the surrounding NLC matrix, the other acting directly on the mesogen.

We acknowledge financial support by MICINN (Project No. FIS2006-03525) and by DURSI (Project No. 2009 SGR 1055). S.H.-N. acknowledges the support from the FPU Fellowship (AP2009-0974). P.T. was supported by the program "Juan de la Cierva". We thank Oriol Güell for providing us with the ellipsoids.

\section{References}

1 G. M. Koenig, I.-H. Lin and N. L. Abbott, Proceedings of the National Academy of Sciences, 2010, 107, 3998-4003.

2 J. S. Lintuvuori, K. Stratford, M. E. Cates and D. Marenduzzo, Physical Review Letters, 2010, 105, 178302.

3 O. D. Lavrentovich, I. Lazo and O. P. Pishnyak, Nature, 2010, 467, $947-$ 950.

4 O. P. Pishnyak, S. Tang, J. R. Kelly, S. V. Shiyanovskii and O. D. Lavrentovich, Physical Review Letters, 2007, 99, 127802.
5 S. Ramaswamy, R. Nityananda, V. Raghunathan and J. Prost, Molecular Crystals and Liquid Crystals, 1996, 288, 175-180.

6 P. Poulin, H. Stark, T. Lubensky and D. Weitz, Science, 1997, 275, 17701773.

7 P. Poulin and D. Weitz, Physical Review E, 1998, 57, 626-637.

8 T. Lubensky, D. Pettey, N. Currier and H. Stark, Physical Review E, 1998, 57, 610-625.

9 J. Loudet, P. Barois and P. Poulin, Nature, 2000, 407, 611-613.

10 J. Yamamoto and H. Tanaka, Nature, 2001, 409, 321-324.

11 M. Yada, J. Yamamoto and H. Yokoyama, Physical Review Letters, 2004, 92, 185501.

12 T. Yamamoto, H. Yokoyama and Y. Tabe, Molecular Crystals and Liquid Crystals, 2007, 478, 211-219.

13 V. G. Nazarenko, A. B. Nych and B. J. Lev, Physical Review Letters, 2001, 87, 075504.

14 I. I. Smalyukh, S. Chernyshuk, B. J. Lev, A. B. Nych, U. Ognysta, V. G. Nazarenko and O. D. Lavrentovich, Physical Review Letters, 2004, 93, 117801.

15 I. Muševič, M. Škarabot, U. Tkalec, M. Ravnik and S. Žumer, Science, 2006, 313, 954-958.

16 M. Ravnik, M. Škarabot, S. Žumer, U. Tkalec, I. Poberaj, D. Babič, N. Osterman and I. Muševič, Physical Review Letters, 2007, 99, 247801.

17 U. Ognysta, A. Nych, V. Nazarenko, I. Muševič, M. Škarabot, M. Ravnik, S. Žumer, I. Poberaj and D. Babič, Physical Review Letters, 2008, 100, 217803.

18 H. Qi, B. Kinkead and T. Hegmann, Advanced Functional Materials, 2008, 18, 212-221.

19 S. Acharya, S. Kundu, J. P. Hill, G. J. Richards and K. Ariga, Advanced Materials, 2009, 21, 989+.

20 I. Muševič and M. Škarabot, Soft Matter, 2008, 4, 195-199.

21 C. Lapointe, A. Hultgren, D. M. Silevitch, E. J. Felton, D. H. Reich and R. L. Leheny, Science, 2004, 303, 652-655.

22 J. B. Rovner, C. P. Lapointe, D. H. Reich and R. L. Leheny, Physical Review Letters, 2010, 105, 228301.

23 U. Tkalec, M. Škarabot and I. Muševič, Soft Matter, 2008, 4, 2402-2409.

24 F. Mondiot, S. P. Chandran, O. Mondain-Monval and J. C. Loudet, Physical Review Letters, 2009, 103, 238303.

25 C. P. Lapointe, T. G. Mason and I. I. Smalyukh, Science, 2009, 326, $1083-$ 1086.

26 C. P. Lapointe, S. Hopkins, T. G. Mason and I. I. Smalyukh, Physical Review Letters, 2010, 105, 178301.

27 I. Lelidis and C. Oedman, Liquid Crystals, 2003, 30, 643-649.

28 P. Oswald and J. Ignés-Mullol, Physical Review Letters, 2005, 95, 027801.

29 P. Tierno, R. Albalat and F. Sagués, SMALL, 2010, 6, 1749-1752.

30 We also used MBBA $\left(\varepsilon_{a}=-0.53\right.$, Synthon $)$ and we observed the same behaviour.

31 P. G. de Gennes and J. Prost, The Physics of Liquid Crystals, Oxford University Press, 1993.

32 P. E. Cladis, Y. Couder and H. R. Brand, Physical Review Letters, 1985, 55, 2945-2948.

33 A. Eremin, C. Bohley and R. Stannarius, European Physical Journal E, 2006, 21, 57-67.

34 M. Nespoulous, C. Blanc and M. Nobili, Physical Review Letters, 2010, 104, 097801.

35 K. S. M. Kojima., J. Yamamoto and K. Yoshikawa, Chemical Physics Letters, 2008, 457, 130-133. 
Supporting Information for the article:

\title{
Breaking the degeneracy of nematic liquid crystals by means of actuated anisometric paramagnetic colloids
}

\author{
S. Hernàndez-Navarro, P. Tierno, J. Ignés-Mullol, F. Sagués,
}

Departament de Química Física, Universitat de Barcelona, Martí i Franques 1, 08028, Spain; Institut de Nanociencia i Nanotecnologia IN²UB, Barcelona, Spain

Email: jignes@ub.edu.

Three movie files are included as supplementary information. The files can be accessed using a web browser following the hyperlinks provided below.

Video 1 (.MPEG1): Relaxation of the target pattern when the magnetic field is turned off. The applied voltage across the cell was $9 \mathrm{~V}$ with a frequency of $1 \mathrm{kHz}$.

http://www.ub.edu/socsam/jordi/movies/admat/video1.mpg

Video 2 (.MPEG1): generation of a helix pattern with a CW orientation. The voltage applied across the cell periodically changes between 2.5 and $6 \mathrm{~V}$ with a period of 1 second. The voltage variations correspond to reversible changes from the target pattern (6 V) to the CW helix (2.5 V) and back. The CW orientation of the helix is due to the CW rotation of the applied magnetic field which generates the target. http://www.ub.edu/socsam/jordi/movies/admat/video2.mpg

Video 3 (.MPEG1): generation of a helix pattern with a CCW orientation. The voltage applied across the cell periodically changes between 2.4 and $6 \mathrm{~V}$ with a period of 3.5 second. The voltage variations correspond to reversible changes from the target pattern (6 V) to the CCW helix (2.4 V) and back. The CCW orientation of the helix is due to the CCW rotation of the applied magnetic field which generates the target.

http://www.ub.edu/socsam/jordi/movies/admat/video3.mpg 